\title{
Modelo econômico para determinação do tempo de reposição na irrigação por sulco
}

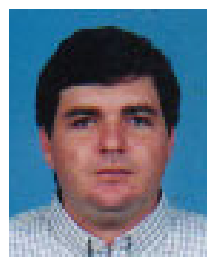

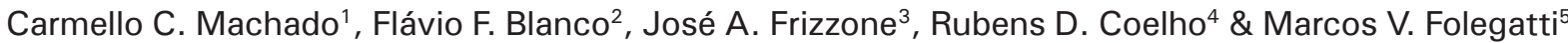 \\ ${ }^{1}$ ESALQ/USP. Av. Pádua Dias 11, CP 9, CEP 13418-900, Piracicaba, SP. Fone: (19) 3429-4217, Ramal 263. \\ E-mail: ccmachad@carpa.ciagri.usp.br (Foto) \\ ${ }^{2}$ ESALQ/USP. Ramal 268. E-mail: ffblanco@carpa.ciagri.usp.br \\ ${ }^{3}$ ESALQ/USP. Ramal 254. E-mail: frizzone@carpa.ciagri.usp.br \\ ${ }^{4}$ ESALQ/USP. Ramal 250. E-mail: rdcoelho@carpa.ciagri.usp.br \\ ${ }^{5}$ ESALQ/USP. Ramal 253. E-mail: mvfolega@carpa.ciagri.usp.br
}

Protocolo 124 - 17/8/2001

\begin{abstract}
Resumo: A utilização de critérios econômicos para tomada de decisão na agricultura, tem sido tema de diversos trabalhos na área de irrigação, proporcionando maior segurança na adoção de práticas de manejo, visando maximizar o retorno econômico. Neste trabalho, apresenta-se uma metodologia para a determinação do tempo de reposição econômico na irrigação por sulco, baseada na otimização da receita líquida, podendo ser aplicada para diferentes culturas, desde que se conheça a função de resposta da cultura à água, a duração de cada fase do ciclo e seus respectivos valores de $\mathrm{Kc}$, assim como os custos envolvidos na produção. O modelo foi aplicado na cultura do tomate para processamento industrial e os resultados demonstraram que a receita líquida máxima foi obtida quando apenas os $70 \mathrm{~m}$ iniciais do sulco receberam a lâmina de irrigação requerida pela cultura, estando os $30 \mathrm{~m}$ restantes sob condições de déficit hídrico, resultando numa receita líquida de $\mathrm{R} \$ 354,00 \mathrm{ha}^{-1}$. A utilização do modelo otimiza a disponibilidade hídrica em condição de tarifação do metro cúbico de água de irrigação.
\end{abstract}

Palavras-chave: tomate industrial, eficiência da irrigação, receita líquida

\section{Economic model for determination of wetting phase time in furrow irrigation}

\begin{abstract}
Using an economic criteria to support agricultural decisions has been the subject of various scientific papers in the irrigation area, providing an increase in security on the adoption of management practices, in order to maximize the return. This work presents a methodology to quantify the economic irrigation time for furrow irrigation, based on the net income optimization. The proposed methodology may be applied for any crop based on: a) the water-yield function for the growing season, b) duration of each phenological phase with respective Kc values, c) information about the costs involved in the cultivation process. The model was applied for processing tomato crop and the results showed that the maximum net income was obtained when only the initial $70 \mathrm{~m}$ of the furrow received the irrigation water depth required by the crop, being the remaining $30 \mathrm{~m}$ under water deficit conditions, resulting in a net income of $\mathrm{R} \$ 354.00 \mathrm{ha}^{-1}$. The use of this model optimizes the water availability when the cubic meter of water is charged.
\end{abstract}

Key words: processing tomato, irrigation efficiency, net income

\section{INTRODUÇ̃̃O}

Os sistemas de irrigação por superfície ocupam, cerca de $60 \%$ da área irrigada no Brasil, e constituem o principal método utilizado em algumas regiões, como Sul e Norte, onde ocupam 93,7 e 97,8\%, respectivamente, da área total irrigada (Telles, 1999). Embora existam inúmeras tecnologias disponíveis para o manejo racional da irrigação, a grande maioria dos produtores ainda irriga de forma inadequada (Marouelli et al., 2000).
Dentre os sistemas de irrigação mais utilizados no Brasil, os de irrigação por superfície apresentam, em geral, os menores custos anuais de operação, incluindo os custos fixos e os variáveis (Scaloppi, 1986). Entretanto, o sistema de irrigação por sulco apresenta baixa eficiência, sendo que apenas $45 \%$ da água derivada ao sulco são efetivamente utilizados pelos cultivos (Paz et al., 2000) tornando-se necessário o desenvolvimento de estratégias de manejo da irrigação que permitam reduzir a quantidade de água aplicada, maximizando a sua eficiência de 
utilização e aumentando a lucratividade, principalmente para regiões em que a água é escassa.

Segundo Raghuwanshi \& Wallender (1998) muitas variáveis influenciam na performance da irrigação por sulco, porém a maior parte não apresenta flexibilidade do ponto de vista de manejo. As variáveis mais flexíveis são a vazão derivada ao sulco, o tempo de corte e a freqüência de irrigação. Segundo Frizzone (1992) maximizar a eficiência nem sempre significa otimizar o lucro, pois o aumento dos custos da irrigação está normalmente associado ao aumento na uniformidade da irrigação. Os benefícios econômicos esperados com a alta eficiência de aplicação devem, portanto, ser cuidadosamente avaliados em relação aos custos associados aos altos índices de uniformidade. De acordo com Cuenca (1989) a otimização de um sistema de irrigação por sulco deve considerar o custo total do sistema de irrigação e os benefícios totais obtidos, sendo estes considerados função do nível de irrigação. Neste caso, o nível de irrigação pode estar relacionado tanto à lâmina de água a ser aplicada durante o ciclo da cultura quanto à extensão de área a ser irrigada.

Para o processamento industrial, o tomate é a hortaliça mais importante da agroindústria brasileira, com crescimento de mercado estimado em $10 \%$ ao ano. A área total cultivada em 1998 foi de aproximadamente 22.280 ha (FNP Consultoria e Comércio, 2001). A água é um dos fatores mais importantes que afetam a produtividade e a qualidade industrial de frutos de tomate (Marouelli et al., 1991; Battilani et al., 1999). Assim, o controle adequado da irrigação constitui fator preponderante para o êxito da cultura, maximizando a produtividade, a eficiência no uso de água e nutrientes e minimizando os custos de irrigação, tornando a atividade mais lucrativa.

Apresenta-se, neste trabalho, uma metodologia para avaliação da viabilidade econômica da irrigação por sulco com drenagem livre, baseada na receita líquida obtida a partir de diferentes tempos de reposição da lâmina d'água no final do sulco. O modelo foi utilizado para determinar o tempo de reposição de água na irrigação por sulco no tomate industrial, buscando-se a maximização da receita líquida ou lucratividade da produção.

\section{MATERIAL E MÉTODOS}

\section{Descrição do modelo}

A metodologia proposta baseia-se no estudo dos índices de eficiência que proporcionam a maior receita líquida no ciclo da cultura. A receita líquida gerada pela irrigação de uma cultura pode ser descrita por:

$$
\begin{gathered}
R B=Y P \\
R L=Y P-C_{r}
\end{gathered}
$$

em que:

$\mathrm{RB}$ - receita bruta, $\mathrm{R} \$ \mathrm{ha}^{-1}$

$\mathrm{Y}$ - produtividade, $\mathrm{kg} \mathrm{ha}^{-1}$

$\mathrm{P} \quad$ - preço de venda, $\mathrm{R} \$ \mathrm{~kg}^{-1}$

$\mathrm{RL}$ - receita líquida, $\mathrm{R} \$ \mathrm{ha}^{-1}$

$\mathrm{C}_{\mathrm{T}}$ - custo total de produção, $\mathrm{R} \$ \mathrm{ha}^{-1}$
A função de custo de produção é dada por:

$$
\mathrm{C}_{\mathrm{r}}=\mathrm{Co}+\mathrm{CWW}
$$

em que:

Co - compõe os custos fixos e variáveis de produção independentes do insumo água, $\mathrm{R} \$ \mathrm{ha}^{-1}$

$\mathrm{Cw}$ - custo total da água, $\mathrm{R} \$ \mathrm{~mm}^{-1} \mathrm{ha}^{-1}$

W - lâmina de água aplicada, $\mathrm{mm}$

A estimativa da produção da cultura é realizada a partir de uma função de resposta da cultura à água, a qual pode ser expressa apenas em função da lâmina de água aplicada, contanto que os demais fatores permaneçam fixos, em níveis ótimos. Geralmente, a função assume a forma quadrática, pois tanto o excesso quanto a limitação de água resultam em perdas de rendimento, estando o ponto de máxima produção em um nível intermediário. A diferencial total desta função igualada a zero fornece a lâmina de água a ser aplicada para máxima produção, que possibilita o cálculo da produção máxima possível de ser obtida durante todo o ciclo da cultura.

Como as exigências em água pela cultura em cada fase de desenvolvimento são diferenciadas, a quantidade total de água a ser aplicada durante todo o ciclo deve ser ponderada para cada uma dessas fases, em função do respectivo coeficiente de cultivo (Kc) para que se possa elaborar uma estratégia de manejo da irrigação. Esta ponderação é obtida por:

$$
\mathrm{W}_{\mathrm{fi}}=\mathrm{Wo} \mathrm{Kc}_{\mathrm{ri}}
$$

donde:

$\mathrm{W}_{\mathrm{fi}}$ - lâmina de água a ser aplicada na fase i, mm

Wo - lâmina para máxima produção, mm

$\mathrm{Kc}_{\mathrm{ri}}$ - coeficiente de cultivo relativo para a fase $\mathrm{i}$

$\mathrm{O}$ valor de $\mathrm{Kc}_{\mathrm{ri}}$ leva em conta o $\mathrm{Kc}$ da fase em questão e a sua duração (em dias), e é estimado por:

$$
K c_{r i}=\frac{N D_{i} K c_{i}}{\sum_{i=1}^{n}\left(N D_{i} K c_{i}\right)}
$$

sendo $\mathrm{ND}_{\mathrm{i}}$ o número de dias da fase $\mathrm{i}, \mathrm{Kc}_{\mathrm{i}}$ o $\mathrm{Kc}$ da fase i e n o número total de fases do ciclo da cultura. De modo geral, o valor calculado de $\mathrm{W}_{\mathrm{fi}}$ é excessivamente grande para ser aplicado em uma única irrigação. Em irrigação por sulco, a lâmina de água a ser aplicada em uma irrigação deve estar entre 30 e $50 \mathrm{~mm}$, pois aplicações de lâminas maiores resultam em altos índices de perda por percolação e escoamento (Frizzone, 1993). Portanto, $\mathrm{W}_{\mathrm{fi}}$ deve ser dividido em determinado número de aplicações que proporcionem lâminas de irrigação dentro desses limites.

O tempo de oportunidade para a infiltração da lâmina requerida é calculado a partir da equação de infiltração acumulada de água no solo e o tempo de avanço da água no sulco pela equação de avanço, até o ponto onde se deseja infiltrar a lâmina requerida. Desta forma, o tempo de reposição pode ser calculado por:

$$
\text { Trep }=\text { Top }+ \text { Tav }- \text { Trec }
$$


em que:

Trep - tempo de reposição, min

Top - tempo de oportunidade, min

Tav - tempo de avanço, min

Trec - tempo de recesso, $\min$

O tempo de recesso deve ser determinado em ensaio de campo, até que a água se infiltre totalmente no final do sulco. Os tempos de recesso nos diferentes pontos do sulco podem ser estimados assumindo-se o recesso linear. Com isto, a análise pode ser realizada para diferentes níveis de déficit ao longo do sulco, devendo-se considerar o tempo de avanço no ponto até o qual se pretende infiltrar a lâmina requerida. Quanto mais próximo do início do sulco for este ponto, maior será o comprimento de sulco que estará recebendo uma lâmina menor que a lâmina requerida e, conseqüentemente, maior será a porcentagem da área na qual as plantas estarão sob condições de déficit hídrico.

Como a lâmina infiltrada em um ponto diminui ao longo do sulco, este foi dividido em trechos e a lâmina infiltrada em cada trecho foi calculada pela equação de infiltração, com base no tempo de oportunidade médio do trecho. A lâmina total infiltrada em cada trecho $\left(\mathrm{LTI}_{t}\right)$ durante todo o ciclo da cultura, é dada por:

$$
\mathrm{LTI}_{\mathrm{t}}=\sum_{\mathrm{i}=1}^{\mathrm{n}}\left(\mathrm{I}_{\mathrm{i}} \mathrm{X}_{\mathrm{i}}\right)
$$

sendo:

$\mathrm{I}_{\mathrm{i}} \quad$ - lâmina de água infiltrada no trecho t em cada evento de irrigação da fase i de desenvolvimento da cultura, $\mathrm{mm}$

$\mathrm{X}_{\mathrm{i}} \quad$ - número de irrigações da referida fase $\mathrm{i}$

Assim, pode-se estimar a produção da cultura em cada trecho, a partir da função de resposta da cultura à água e a receita bruta pode ser calculada.

Conhecendo-se os custos de produção por hectare cultivado e o custo cobrado pela utilização da água, determina-se a função de custo e se pode calcular a receita líquida para cada trecho do sulco, da seguinte forma:

$$
\mathrm{RL}_{\mathrm{t}}=(\mathrm{YP}-\mathrm{Co}-\mathrm{CW} \mathrm{W}) \frac{\mathrm{C}_{\mathrm{t}}}{\mathrm{C}_{\mathrm{T}}}
$$

em que:

$\mathrm{RL}_{\mathrm{t}}$ - receita líquida para o trecho t, $\mathrm{R} \$$ /área do trecho em ha

$\mathrm{C}_{\mathrm{t}}$ - comprimento do trecho $\mathrm{t}, \mathrm{m}$

$\mathrm{C}_{\mathrm{T}}$ - comprimento total do sulco, $\mathrm{m}$

A receita líquida total é dada por:

$$
\mathrm{RL}_{\mathrm{T}}=\sum_{\mathrm{t}=1}^{\mathrm{n}}\left(\mathrm{RL}_{\mathrm{t}}\right) \frac{10000}{\mathrm{C}_{\mathrm{T}} \mathrm{E}}
$$

sendo $\mathrm{RL}_{\mathrm{T}}$ a receita líquida total, $\mathrm{R} \$ \mathrm{ha}^{-1}$, n o número de trechos e E o espaçamento entre sulcos, $\mathrm{m}$.

\section{Aplicação do modelo}

A função de resposta do tomateiro à água utilizada no modelo foi desenvolvida para os perímetros irrigados da cidade de Petrolina, PE (Dantas Neto, 1994) sendo:

$$
Y=-0,2395 W^{2}+271,94 W-23000
$$

em que:

Y - produtividade da cultura, $\mathrm{kg} \mathrm{ha}^{-1}$

W - lâmina total de água aplicada durante o ciclo, $\mathrm{mm}$

A lâmina de irrigação obtida para máxima produção foi de $568 \mathrm{~mm}$, assumida como sendo a lâmina requerida pela cultura durante o ciclo, resultando em uma produtividade de $54.197,4 \mathrm{~kg} \mathrm{ha}^{-1}$.

A duração de cada fase de desenvolvimento da cultura e o respectivo Kc utilizados, são apresentados por Doorenbos \& Kassam (1994); daí, calculou-se a lâmina de água a ser aplicada em cada fase, que foram divididas em determinado número de irrigações, de modo que a lâmina aplicada em cada evento de irrigação estivesse dentro dos limites de 30 a 50 mm (Tabela 1).

Tabela 1. Dados de entrada utilizados no modelo para a determinação do tempo de reposição econômico na irrigação por sulco, no tomate industrial

\begin{tabular}{ccccccc} 
Fase $^{*}$ & $\begin{array}{c}\text { NDI } \\
\text { (dias) }\end{array}$ & $\mathrm{Kc}$ & $\mathrm{Kc}_{\mathrm{ri}}$ & $\mathrm{W}_{\mathrm{fi}}$ & $\mathrm{X}_{\mathrm{i}}$ & $\begin{array}{c}\text { Lâmina por } \\
\text { Irrigação } \\
(\mathrm{mm})\end{array}$ \\
\hline DV & 22 & 0,75 & 0,21 & 119,9 & 3 & 40,0 \\
FL & 25 & 1,15 & 0,37 & 208,9 & 5 & 41,8 \\
FC & 25 & 0,87 & 0,28 & 158,1 & 4 & 39,5 \\
MT & 18 & 0,62 & 0,14 & 81,1 & 2 & 40,6 \\
\hline * DV:
\end{tabular}

Utilizaram-se, nas análises, sulcos de $100 \mathrm{~m}$ de comprimento e espaçados de $1 \mathrm{~m}$, com vazão derivada ao sulco igual a $0,8 \mathrm{~L} \mathrm{~s}^{-1}$. As equações de avanço (Tx) e de infiltração acumulada (I) utilizadas foram aquelas determinadas por Garrido et al. (1994) para um solo de textura média, apresentadas a seguir:

$$
\begin{aligned}
& \mathrm{TX}=4,265 \mathrm{X}^{0,689} \\
& \mathrm{I}=0,95116 \mathrm{~T}^{0,393}
\end{aligned}
$$

sendo Tx o tempo de avanço na distância $\mathrm{x}$, min, $\mathrm{X}$ a distância em relação ao início do sulco, m, I a infiltração acumulada, m, e T o tempo de infiltração, min.

$\mathrm{O}$ valor de venda do produto $(\mathrm{P})$ foi de $\mathrm{R} \$ 80,00 \mathrm{t}^{-1} \mathrm{e}$ os custos de produção independentes da água (Co) de $\mathrm{R} \$ 2.900,00 \mathrm{ha}^{-1}$. $\mathrm{O}$ custo total da água $(\mathrm{Cw})$ foi dado pela soma dos custos de utilização da água, manutenção do sistema, energia elétrica e mão-de-obra para o manejo da irrigação, sendo $\mathrm{Cw}$ igual a $\mathrm{R} \$ 0,129 \mathrm{~m}^{-3}$.

As análises foram feitas para diferentes comprimentos de sulco com déficit, em intervalos de $10 \mathrm{~m}$, até que $50 \%$ do comprimento do sulco estivessem sendo irrigados com uma lâmina menor que a requerida. 


\section{RESULTADOS E DISCUSSÃO}

Os resultados da análise econômica e os tempos de reposição de água no sulco para as diferentes fases do ciclo da cultura, são apresentados na Tabela 2. Verifica-se a tendência de aumento na receita líquida (RL) com o aumento da área de déficit (redução da distância sem déficit) devido à economia proporcionada pela aplicação de um volume menor de água, porém o aumento da RL não é indefinido, pois esta depende dos custos de produção, do preço de venda do produto e do custo de aplicação de água. Observa-se que a RL variou de $\mathrm{R} \$ 66,00 \mathrm{ha}^{-1}$ para a condição de irrigação sem déficit a $\mathrm{R} \$ 354,00 \mathrm{ha}^{-1}$ para a condição em que apenas os $70 \mathrm{~m}$ iniciais do sulco receberam a lâmina requerida pela cultura. Portanto, este é o ponto de máxima eficiência do sistema, onde a receita líquida é máxima, como pode ser observado na Figura 1. A produtividade média na área obtida para este esquema de irrigação foi de $51.266 \mathrm{~kg} \mathrm{ha}^{-1}$.

Tabela 2. Receita líquida, receita líquida relativa, e tempo de reposição de água no sulco por irrigação para cada fase do ciclo da cultura do tomate industrial, para diferentes condições de déficit hídrico simuladas

\begin{tabular}{|c|c|c|c|c|c|c|}
\hline \multirow{3}{*}{$\begin{array}{l}\text { Distância } \\
\text { sem Déficit } \\
\text { (m) }\end{array}$} & \multirow{3}{*}{$\begin{array}{l}\text { Receita } \\
\text { Líquida } \\
\left(\mathrm{R} \$ \mathrm{ha}^{-1}\right)\end{array}$} & \multirow{3}{*}{$\begin{array}{l}\text { Receita } \\
\text { Líquida } \\
\text { Relativa }\end{array}$} & \multirow{2}{*}{\multicolumn{4}{|c|}{$\begin{array}{c}\text { Tempo de Reposição (min) } \\
\text { Fase do Ciclo }^{*}\end{array}$}} \\
\hline & & & & & & \\
\hline & & & $\mathrm{DV}$ & FL & $\mathrm{FC}$ & MT \\
\hline 100 & 66 & 0,091 & 8,6 & 13,2 & 7,5 & 10,0 \\
\hline 90 & 205 & 0,284 & 11,6 & 16,2 & 10,5 & 13,0 \\
\hline 80 & 298 & 0,412 & 14,6 & 19,2 & 13,5 & 16,0 \\
\hline 70 & 354 & 0,489 & 17,6 & 22,2 & 16,5 & 19,0 \\
\hline 60 & 342 & 0,473 & 20,6 & 25,2 & 19,5 & 22,0 \\
\hline 50 & 163 & 0,225 & 23,6 & 28,2 & 22,5 & 25,0 \\
\hline
\end{tabular}

* DV: desenvolvimento vegetativo; FL: floração; FC: formação da colheita; MT: maturação

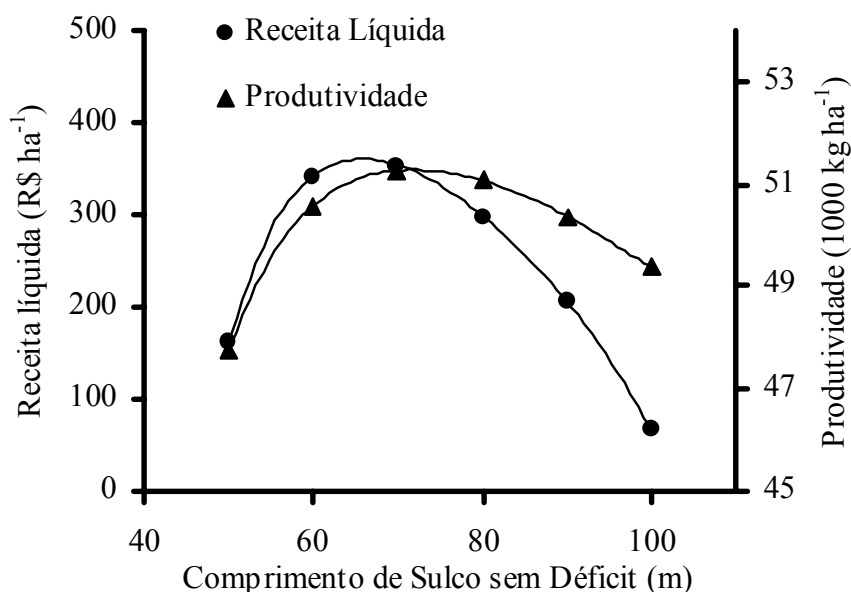

Figura 1. Receita líquida e produtividade média obtidas em função do comprimento de sulco sem déficit para a cultura do tomate industrial

Os tempos de reposição que proporcionaram maior receita líquida foram 17,6 min para o período vegetativo, 22,2 min para a fase de floração, 16,5 min para a fase de formação da colheita e 19,0 min para a fase de maturação. Os tempos de corte para cada fase do ciclo (Tabela 3) que resultou na maior receita líquida, foram de 92,2, 101,9,96,1 e 98,7 min para as fases vegetativa, floração, formação da colheita e maturação, respectivamente, representando redução de mais ou menos $12 \%$ no tempo de corte, em relação à condição em que a lâmina de água requerida pela cultura foi aplicada em toda a extensão do sulco (distância sem déficit igual a 100 m).

Tabela 3. Tempo de corte por irrigação para cada fase do ciclo do tomate industrial para diferentes condições de déficit hídrico simuladas e o tempo total de irrigação, durante o ciclo da cultura

\begin{tabular}{crrrrc}
\hline \multirow{2}{*}{$\begin{array}{c}\text { Distância } \\
\text { sem Déficit } \\
(\mathrm{m})\end{array}$} & \multicolumn{3}{c}{ Tempo de Corte (min) } & \multirow{2}{*}{$\begin{array}{c}\text { Tempo Total de } \\
\text { Irrigação no Ciclo }\end{array}$} \\
\cline { 2 - 4 } & \multicolumn{1}{c}{ DV } & \multicolumn{1}{c}{ FL } & \multicolumn{1}{c}{ FC } & \multicolumn{1}{c}{ MT } & (min) \\
\hline 100 & 110,4 & 115,0 & 109,3 & 111,9 & 1568 \\
90 & 106,3 & 110,9 & 105,2 & 107,7 & 1510 \\
80 & 101,9 & 106,5 & 100,8 & 103,4 & 1448 \\
70 & 97,2 & 101,9 & 96,1 & 98,7 & 1383 \\
60 & 92,2 & 96,8 & 91,1 & 93,7 & 1313 \\
50 & 86,8 & 91,4 & 85,7 & 88,2 & 1236 \\
\hline
\end{tabular}

* DV: desenvolvimento vegetativo; FL: floração; FC: formação da colheita; MT: maturação

Em relação à condição sem déficit, o ponto ótimo proporcionou redução do volume total de água aplicado durante o período de cultivo, que resultou numa economia de $886 \mathrm{~m}^{3}$ de água, havendo redução das perdas por percolação e escoamento, de $\mathrm{R} \$ 234,90$ para $\mathrm{R} \$ 159,90 \mathrm{ha}^{-1}$ (Tabela 4). Considerando-se uma área de 50 ha plantada com o tomate industrial, o manejo da irrigação com déficit em $30 \%$ da área proporcionaria uma economia de $44.300 \mathrm{~m}^{3} \mathrm{de}$ água, e $\mathrm{R} \$ 3.750,00$ deixariam de ser gastos com as perdas por percolação e escoamento superficial durante um ciclo de cultivo; este procedimento não traria apenas benefícios ao produtor, devido à redução dos custos de produção mas, também, um ganho para o agroecossistema, visto que a escassez de água se agrava ao longo dos anos e a preservação desse recurso é fundamental para o desenvolvimento de uma agricultura sustentável.

Tabela 4. Volume de água aplicado (Apl), infiltrado (Inf), percolado (Per) e escoado (Esc) e o custo com as perdas por percolação e escoamento durante o ciclo do tomate industrial irrigado por sulco, para as diferentes condições de déficit hídrico simuladas

\begin{tabular}{|c|c|c|c|c|c|c|}
\hline \multirow{3}{*}{$\begin{array}{l}\text { Distância } \\
\text { sem Déficit } \\
(\mathrm{m})\end{array}$} & \multicolumn{4}{|c|}{ Volume } & \multicolumn{2}{|c|}{ Custo } \\
\hline & Apl & Inf & Per & Esc & Per & Esc \\
\hline & \multicolumn{4}{|c|}{$\mathrm{m}^{3} \mathrm{ha}^{-1}$} & \multicolumn{2}{|c|}{$\mathrm{R} \$ \mathrm{ha}^{-1}$} \\
\hline 100 & 7524 & 6883 & 1187 & 641 & 153,2 & 81,7 \\
\hline 90 & 7244 & 6631 & 968 & 613 & 124,9 & 78,6 \\
\hline 80 & 6950 & 6345 & 785 & 605 & 101,3 & 77,3 \\
\hline 70 & 6638 & 6016 & 624 & 622 & 80,5 & 79,4 \\
\hline 60 & 6302 & 5619 & 487 & 683 & 62,9 & 86,9 \\
\hline 50 & 5936 & 5094 & 367 & 842 & 47,4 & 107,5 \\
\hline
\end{tabular}

O incremento de receita líquida, utilizando-se os tempos de reposição correspondentes ao comprimento sem déficit de $70 \mathrm{~m}$, em relação àqueles referentes ao comprimento de $100 \mathrm{~m}$, foi de $\mathrm{R} \$ 288,00 \mathrm{ha}^{-1}$. Marouelli et al. (2000) obtiveram valores de incremento de receita líquida na produção do tomate para processamento industrial, em torno de $\mathrm{R} \$ 230,00 \mathrm{ha}^{-1}$ para a cultura irrigada por pivô central, e de $\mathrm{R} \$ 270,00$ ha $^{-1}$ para aspersão convencional para áreas acima de $10 \mathrm{ha}$, quando se utilizou o manejo racional da irrigação, porém a receita líquida obtida foi de R $\$ 1.000,00 \mathrm{ha}^{-1}$ para aspersão convencional, e de 
$\mathrm{R} \$ 1.400,00 \mathrm{ha}^{-1}$ para pivô central, superando a receita líquida obtida nesse estudo, que foi de $\mathrm{R} \$ 354,00 \mathrm{ha}^{-1}$. Esta diferença deve estar relacionada à não cobrança pela utilização da água, bem como às maiores produtividades alcançadas por esses autores: $69 \mathrm{t} \mathrm{ha}^{-1}$ para aspersão convencional e de $75 \mathrm{t} \mathrm{ha}^{-1}$ para pivô central.

A eficiência de armazenamento (Es) e a uniformidade de distribuição de água (UD) diminuíram com o aumento da área irrigada com déficit, enquanto a eficiência de aplicação (Ea) aumentou (Figura 2). O aumento de Ea, dado pelo quociente entre o volume útil de água armazenado no solo e o volume aplicado, expressa uma utilização da água de maneira mais racional, visto que uma fração maior do volume de água aplicado ficou retida no solo, disponível para as plantas. Os valores de Es, UD e Ea para a situação que proporcionou a maior receita líquida (70 $\mathrm{m}$ sem déficit) foram, respectivamente, $94,7,78,8$ e $81,1 \%$, os quais são considerados aceitáveis, por Frizzone (1993).

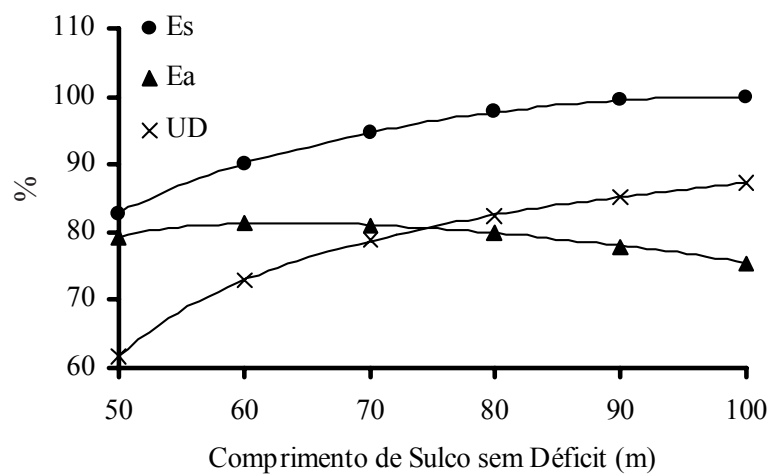

Figura 2. Eficiência de armazenamento (Es) e de aplicação (Ea) e uniformidade de distribuição (UD) da irrigação por sulco no tomate industrial, para as diferentes condições de déficit hídrico simuladas

A análise econômica da irrigação pela metodologia proposta, para determinada cultura, é realizada utilizando-se uma função de produção da cultura à água e o custo pela utilização da água, os quais, geralmente, não são considerados em outras metodologias; assim, esta apresenta grande potencial para a definição da melhor estratégia de irrigação, racionalizando a utilização da água e proporcionando maior lucratividade ao produtor, principalmente nos perímetros irrigados onde a cobrança pela utilização dos recursos hídricos já se faz presente.

\section{CONCLUSÕES}

Para as condições impostas nas simulações, concluiu-se que:

1. Na irrigação por sulco do tomate industrial, a irrigação com déficit é vantajosa, proporcionando aumento substancial na receita líquida, devido à redução do volume total de água aplicado durante o ciclo da cultura.
2. A irrigação plena aplicada apenas nos $70 \mathrm{~m}$ iniciais do sulco, ficando os $30 \mathrm{~m}$ restantes sob condições de déficit hídrico, proporcionou uma receita líquida de $\mathrm{R} \$ 354,00 \mathrm{ha}^{-1} \mathrm{e}$ produtividade de $51.266 \mathrm{~kg} \mathrm{ha}^{-1}$.

\section{LITERATURA CITADA}

Battilani, A.; Piva, A.; Dadomo, M. Evaluation of the decision support system for irrigation scheduling of processing tomato adopted in the Emilia-Romagna region. Acta Horticulturae, The Hague, n.487, p.507-511, 1999.

Cuenca, R.H. Irrigation system design: An engineering approach. New Jersey: Prentice Hall, 1989. 551p.

Dantas Neto, J. Modelos de decisão para otimização do padrão de cultivo, em áreas irrigadas, baseados nas funções de resposta das culturas à água. Botucatu: UNESP, 1994. 125p. Tese Doutorado

Doorenbos, J.; Kassam, A.H. Efeito da água no rendimento das culturas. Campina Grande: UFPB, 1994. 306p. FAO. Estudos de Irrigação e Drenagem, 33

FNP Consultoria e Comércio. Agrianual 2000: anuário da agricultura brasileira. São Paulo: Argos Comunicação, 2001. 546p.

Frizzone, J.A. Irrigação por aspersão: uniformidade e eficiência. Piracicaba: ESALQ - Depto. Engenharia Rural, 1992. 53p. Série Didática, 003

Frizzone, J.A. Irrigação por superfície. Piracicaba: ESALQ Depto. Engenharia Rural, 1993. 183p. Série Didática, 005

Garrido, M.A.T.; Silva, A.M.; Faria, M.A.; Lima, L.A. Estudo da infiltração e avanço de água em sulcos de irrigação sob diferentes regimes de fluxo e vazões. Ciência e Prática, Lavras, v.18, n.2, p.200-208, 1994.

Marouelli, W.A.; Silva, H.R.; Oliveira, C.A.S. Produção de tomate industrial sob diferentes regimes de umidade no solo. Pesquisa Agropecuária Brasileira, Brasília, v.26, n.9, p.1531-1537, 1991.

Marouelli, W.A.; Silva, W.L.C.; Silva, H.R.; Vilela, N.J. Eficiência econômica do manejo racional da irrigação em tomateiro para processamento industrial. Horticultura Brasileira, Brasília, v.18, n.3, p.238-243, 2000.

Paz, V.P.S.; Teodoro, R.E.F.; Mendonça, F.C. Recursos hídricos, agricultura irrigada e meio ambiente. Revista Brasileira de Engenharia Agrícola e Ambiental, Campina Grande, v.4, n.3, p.465-473, 2000.

Raghuwanshi, N.S.; Wallender, W.W. Optimal furrow irrigation scheduling under heterogeneous conditions. Agricultural Systems, Barking Essex, v.58, n.1, p.39-55, 1998.

Scaloppi, E.J. Características dos principais sistemas de irrigação. ITEM, Brasília, n.25, p.22-27, 1986.

Telles, D.A. Água na agricultura e pecuária. In: Rebouças, A.C.; Braga, B.; Tundisi, J.G. (org.) Águas doces no Brasil: capital ecológico, uso e conservação. São Paulo: Escrituras, 1999. cap.9, p.305-338. 